\section{Large-Vessel Giant Cell Arteritis Presenting as Back Pain with Vertebral Body Infarction in a 73-year-old Caucasian Woman}

\section{To the Editor:}

Giant cell arteritis (GCA) is a chronic vasculitis that almost exclusively afflicts individuals age 50 years and older, with a prevalence of 1 in 500 individuals $^{1,2}$. Early diagnosis is always the goal, so symptoms such as headache, scalp pain, jaw claudication, and amaurosis fugax should alert clinicians of the possibility of GCA and lead to early confirmatory biopsy ${ }^{3}$. GCA is also known to occasionally present in an unusual manner, which can cause significant confusion and ultimately delay the diagnosis, as was the case with our patient.

A 73-year-old Caucasian woman with a history of lower back pain secondary to degenerative disc disease presented to the emergency department with a one-week history of incapacitating back and neck pain accompanied by stiffness. She described the pain as sharp, beginning in her mid-back, and then slowly progressing to both her lower cervical area and her upper sacrum. She could not find a position that was comfortable, and any movement made her symptoms much worse. She also complained of "shooting" pains in her forearms bilaterally, with transient numbness of her thumbs, as well as increasing stiffness in both shoulders. She specifically denied any jaw claudication, headaches, or visual disturbances. She had had a one-year history of lumbar degeneration for which she underwent a lumbar fusion of the L5 to S1 vertebrae 4 months prior to presentation. The medical history included known C4 through C6 spinal stenosis, a herpes zoster outbreak earlier that year, gastroesophageal reflux disease, mild interstitial pulmonary fibrosis, and hyperlipidemia. When her symptoms began, she saw her neurosurgeon, who started pulse methylprednisolone and opiates for a presumed cervical radiculopathy. Her symptoms worsened despite this treatment, and she subsequently was seen and released by the emergency department (ED) 3 times over the course of the week prior to admission. During each ED visit, her pain was treated with high-strength opioids. After several days of treatment failure, her neurosurgeon considered the possibility of polymyalgia rheumatica based on her history of increasing shoulder stiffness and an erythrocyte sedimentation rate (ESR) of 102 $\mathrm{mm} / \mathrm{h}$, and he placed her on $20 \mathrm{mg}$ oral prednisone daily. She took this medication for several days and her shoulder stiffness did improve, but her sharp back pain worsened and she presented a fourth time to the ED, when she was admitted to the Internal Medicine service.

Clinical examination at the time of admission was significant for severe neck and back pain with any movement of the cervical and thoracic vertebrae. There was no sensory loss detected in any dermatome. She had intact visual fields bilaterally, nontender temporal arteries, and equal peripheral pulses. Blood pressure was $150 / 80 \mathrm{~mm} \mathrm{Hg}$ in both arms, with no tachycardia or tachypnea on examination. Lung fields were clear and she had no cardiac murmurs or rubs. The rest of the clinical examination was unrevealing. Laboratory studies revealed an ESR of $102 \mathrm{~mm} / \mathrm{h}$ and an elevated antineutrophil antibody at 3.2 units (normal 0.0 to 1.0). Rheumatoid factor (RF), antibodies to cyclic citrullinated peptide (anti-CCP), classical and protoplasmic antineutrophil cytoplasmic antibodies (p-ANCA, c-ANCA), and rapid plasma reagin test were unremarkable. A cervical, thoracic, and lumbar magnetic resonance imaging (MRI) scan was immediately obtained to assess for vertebral fracture or epidural abscess. The MRI revealed edema in the left lateral half of T6-T8 vertebral bodies consistent with infarction (Figure 1). The descending aorta also appeared abnormal on this study and dissection or plaque rupture could not be ruled out. Based on these findings, a dedicated computed tomography (CT) angiogram of the descending aorta was done. The CT revealed no aortic dissection, but did reveal mild circumferential aortic thickening. A transesophageal echocardiogram and MRI angiogram of the descending aorta were done and both confirmed the presence of generalized aortitis (Figure 2).

While her acute pain was controlled with intravenous (IV) narcotics, she was started on $200 \mathrm{mg}$ daily IV methylprednisolone twice daily (total daily dose $400 \mathrm{mg}$ ). After 24 hours of treatment, her pain was reported to

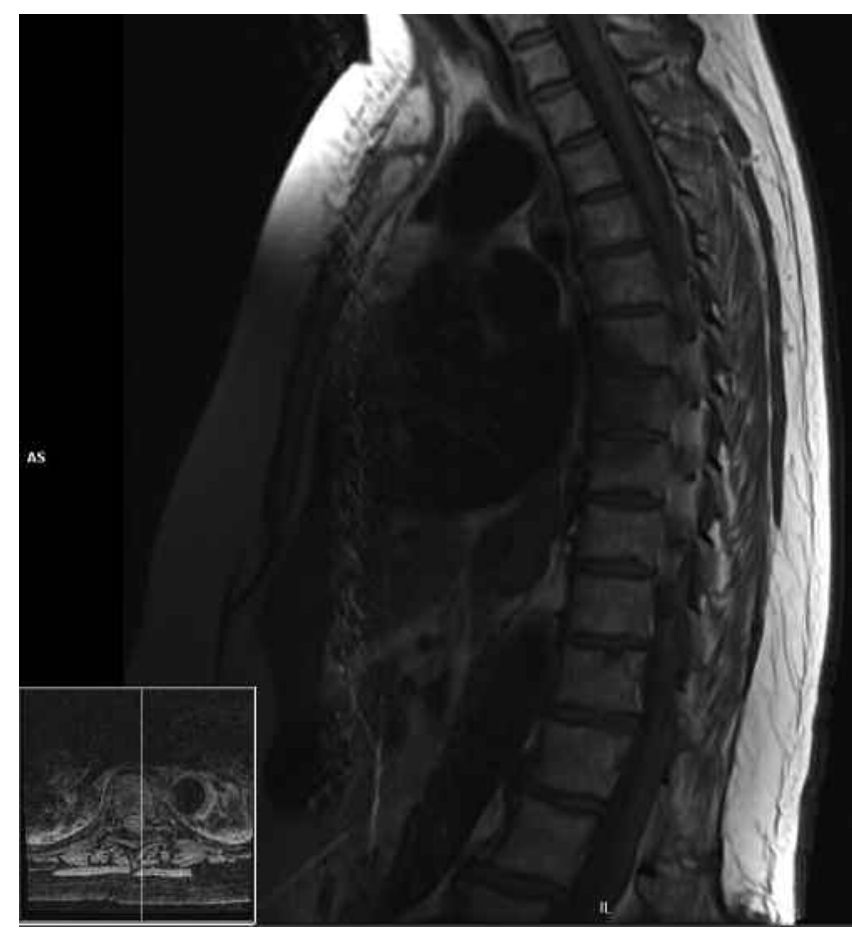

Figure 1. Left lateral-half edema of the T6, T6, and T8, representing acute vertebral bone infarction.

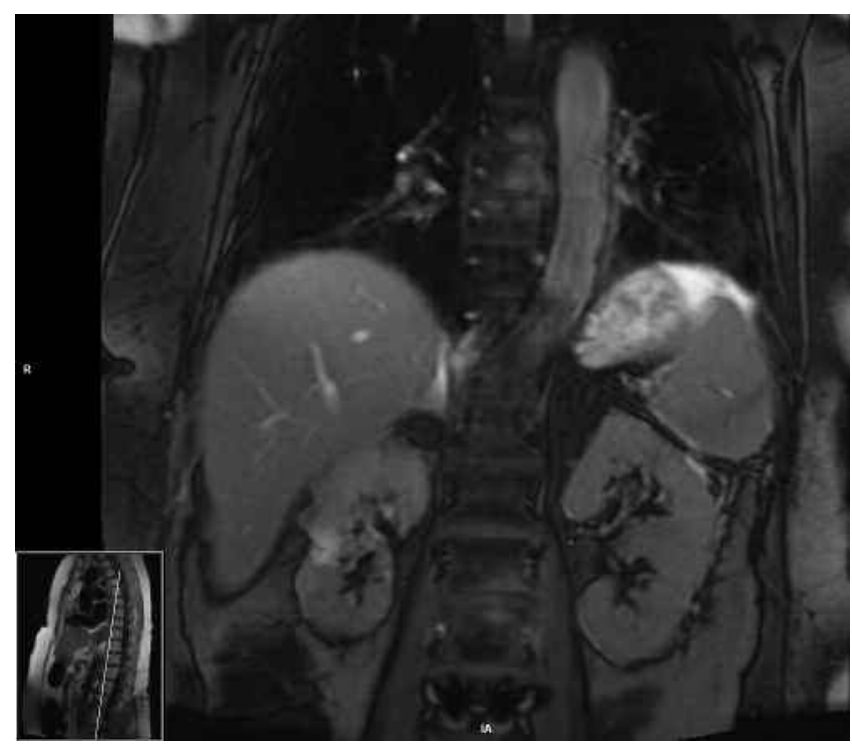

Figure 2. Aorta enhancement.

have decreased by $50 \%$. After 2 days of IV methylprednisolone, she was transitioned to $80 \mathrm{mg}$ prednisone daily. Her ESR dropped from $120 \mathrm{~mm} / \mathrm{h}$ to $38 \mathrm{~mm} / \mathrm{h}$ over the course of 3 days. During the investigations and treatment course, she continued to deny any visual disturbances, jaw claudication, or fevers. Within 5 days of starting high-dose steroids, her cervical and upper thoracic pain as well as her shoulder stiffness had completely abated. There was still some residual pain over the area of the infracted lower thoracic vertebrae. Followup appointments with her primary care physician showed that the symptoms had completely resolved, and she is currently in the process of a steroid taper.

Personal non-commercial use only. The Journal of Rheumatology Copyright @ 2011 . All rights reserved. 
The American College of Rheumatology has published classification criteria for GCA in order to distinguish it from other forms of vasculitis, and our patient meets 2 of the 5 recognized criteria (age of onset $>50$ years, new onset of localized headache, temporal artery tenderness or decreased temporal artery pulse, elevated ESR $\geq 50 \mathrm{~mm} / \mathrm{h}$, and biopsy showing necrotizing arteritis) for diagnosis ${ }^{4}$. Our patient met none of the criteria for Takayasu's arteritis (age of onset $<40$ years, claudication of the extremities, decreased brachial artery pressure, difference in arm blood pressure $>10 \mathrm{~mm} \mathrm{Hg}$, audible subclavian bruit, and global large-vessel narrowing on angiogram), which is the other leading cause of aortitis in adults $^{5}$. Although GCA and Takayasu's arteritis are similar in expression, they are easily separated on the basis of clinical and demographic findings ${ }^{6}$. Although classically affecting the arteries arising from the aortic arch, in particular the temporal and ophthalmologic arteries, in our case there was no known cranial involvement, but severe vasculitis causing vertebral bone infarction in the thoracic spine. While aortitis may present clinically in $3 \%$ to $18 \%$ of temporal arteritis patients, a recent study showed that aortic involvement may be present in over $50 \%$ of cases when imaging with positron emission tomography and MRI was used ${ }^{7}$. While complications such as aneurysms, vascular dissection, strokes, myocardial infarction, and even mesenteric ischemia have been described, GCA causing vertebral body infarction is a previously unreported complication ${ }^{8}$. Prior reports of patients with thoracic vertebral body infarction were associated with spinal cord infarction as well ${ }^{9}$. This would be due to the fact that both the thoracic spinal cord and thoracic vertebrae share the same arterial blood supply from the branches of the posterior intercostal arteries. To our knowledge, there has not been a report of an aortic insult that caused damage to the thoracic vertebrae alone. These infarctions were likely the cause of her lower thoracic back pain, which lingered longer after treatment when compared to her cervical and upper thoracic pain, which was more likely due to the aortitis itself. We chose not to do a temporal artery biopsy for confirmation as the clinical, imaging, and laboratory data as well as the patient's quick response to treatment strongly supported diagnosis of GCA over any other. It is important to note that temporal artery biopsy is less likely to be positive in patients with large-vessel involvement ${ }^{10}$. In this patient, the ultimate diagnosis of GCA was confounded by the lack of response to outpatient low-dose oral pulse methylprednisolone. However, once started on high-dose methylprednisolone (200 mg twice daily) her recovery was swift and profound, consistent with the usual response to corticosteroids.

Physicians treating elderly patients presenting with incapacitating back pain and/or severe movement-induced pain should include vertebral infarction related to large-vessel GCA in their differential diagnosis.
RYAN E. PECK, MD, Mayo School of Graduate Medical Education; MICHAEL J. MANIACI, MD, Division of Hospital Internal Medicine, Mayo Clinic, Jacksonville, Florida, USA. Address correspondence to Dr. Maniaci; E-mail: maniaci.michael@mayo.edu

\section{REFERENCES}

1. Wang X, Hu ZP, Lu W, Tang XQ, Yang HP, Zeng LW, et al. Giant cell arteritis. Rheumatol Int 2008;29:1-7.

2. Lawrence RC, Helmick CG, Arnett FC, Deyo RA, Felson DT, Giannini EH, et al. Estimates of the prevalence of arthritis and selected musculoskeletal disorders in the United States. Arthritis Rheum 1998;41:778-99.

3. Dasgupta B. The Giant Cell Arteritis Guideline Development Group. Concise guidance: diagnosis and management of giant cell arteritis. Clin Med 2010;10:381-6.

4. Hunder GG, Bloch DA, Michel BA, Stevens MB, Arend WP, Calabrese LH, et al. The American College of Rheumatology 1990 criteria for classification of giant cells arteritis. Arthritis Rheum 1990;33:1122-8.

5. Arend WP, Michel BA, Bloch DA, Hunder GG, Calabrese LH, Edworthy SM, et et al. The American College of Rheumatology 1990 criteria for the classification of Takayasu arteritis. Arthritis Rheum 1990;33:1129-34.

6. Arend WP, Michel BA, Hunder, GG. Clinical differentiation between giant cell (temporal) arteritis and Takayasu's arteritis. J Rheumatol 1996;23:106-11.

7. Bossert M, Prati C, Balblanc JC, Lohse A, Wendling D. Aortic involvement in giant cell arteritis: Current data. Joint Bone Spine 2011;78:246-51.

8. Marie I, Proux A, Duhaut P, Primard E, Lahaxe L, Girszyn N, et al. Long-term follow-up of aortic involvement in giant cell arteritis: a series of 48 patients. Medicine (Baltimore) 2009;88:182-92.

9. Suzuki T, Kawaguchi S, Takebayashi T, Yokogushi K, Takada $\mathrm{J}$, Yamashita T. Vertebral body ischemia in the posterior spinal artery syndrome: case report and review of the literature. Spine 2003;28:E260-4.

10. Brack A, Martinez-Taboada V, Stanson A, Goronzy JJ, Weyand CM. Disease pattern in cranial and large-vessel giant cell arteritis. Arthritis Rheum 1999;42:311-7.

J Rheumatol 2011;38:10; doi:10.3899/jrheum.110191 